Pacific Journal of Mathematics

UNIFORM CONVERGENCE FOR MULTIFUNCTION 


\title{
UNIFORM CONVERGENCE FOR MULTIFUNCTIONS
}

\author{
R. E. SMIthsoN
}

\begin{abstract}
Let $\mathscr{F}$ be a collection of multivalued functions on a topological space into uniform space. The topology of uniform convergence is defined on $\mathscr{F}$, and it is shown that for point compact functions this topology is larger than the pointwise topology. Some results are given on uniform convergence of nets in $\mathscr{F}$. It is also shown that if $\mathscr{F}$ consists of point compact continuous functions on a compact space, then the compact open topology and topology of uniform convergence are the same. Finally the following Ascoli theorem for multifunctions is obtained. Theorem: Let $\mathscr{C}$ be the set of point compact, continuous multifunctions on a compact regular space into a $T_{2}$-uniform space. Then $\mathscr{F} \subset \mathscr{C}$ is compact if and only if (i) $\mathscr{F}$ is closed in $\mathscr{C}$, (ii) $\mathscr{F}[x]$ has compact closure for each $x$ and (iii) $\mathscr{F}$ is equicontinuous.
\end{abstract}

1. Introduction. In [2] the topology of pointwise convergence and the compact open topologies were defined for sets of multivalued functions. Basic properties of these topologies (such as separation axioms, etc.) were studied and some characterizations of compact sets were obtained. The purpose of the present paper is to continue the development of the basic topologies to topologies generated by uniformities, and hence, to a discussion of uniform convergence.

Thus let $(X, \mathscr{T})$ be a topological space, and let $(Y, \mathscr{Y})$ be a uniform space. Let $\mathscr{F}$ be a family of multivalued functions on $X$ into $Y$. We construct a uniformity for $\mathscr{F}$ as follows. If $V \in \mathscr{C}$, define $W(V)$ by: $W(V)=\{(F, G) \in \mathscr{F} \times \mathscr{F} \mid$ for all $x \in X, \quad(y, G(x)) \cap V \neq \phi$ for all $y \in F(x)$, and $\left(F(x), \mathrm{y}^{\prime}\right) \cap V \neq \phi$ for all $\left.\mathrm{y}^{\prime} \in G(x)\right\}$.

The cumbersomeness of this definition is a result of the fact that $F(x)$ and $G(x)$ are subsets of $Y$ rather than points. Note, that if $F$ and $G$ are singlevalued functions, then $W(V)=\{(F, G) \mid(F(x), G(x)) \in$ $V\}$. We could have used the set $\{(F, G) \mid F(x) \times G(x) \subset V\}$, but then we might not get many pairs in members of the uniformity. Another possibility is sets of the form $\{(F, G) \mid F(x) \times G(x) \cap V \neq \phi\}$. This could give a reasonable definition, but this condition is somewhat weaker than the one chosen, and in some cases allows too many pairs $(F, G)$ in the set. Now we let $\mathcal{W}$ be the uniformity generated by the collection of all such sets $W(V)$. The topology generated by Wo is called the topology of uniform convergence (we sometimes abbreviate this to the u.c. topology or simply u.c.) and we obtain the following relationship between this topology and the topology of pointwise convergence defined in [2]. 
Definition. A multifunction $F: X \rightarrow Y$ on a topological space $X$ into a topological space $Y$ is called point compact if and only if $F(x)$ is compact for each $x \in X$.

If $(Y, \mathscr{Y})$ is a uniform space, we shall assume that $Y$ has the topology generated by the uniformity $\mathscr{V}$. (See Kelley [1] for notation and definitions.) Further, if $A \subset X$, then $A^{*}$ and $A^{\circ}$ denote the closure and interior of $A$ respectively. Finally, in this paper the terms function and multifunction will be synonymous.

LEMMA 1. If each member of $\mathscr{F}$ is point compact, then the topology of uniform convergence is larger than the topology of pointwise convergence.

Proof. From [2] we have that the pointwise topology is generated by sets of the form $\{F \in \mathscr{F} \mid F(x) \subset U\}$ or $\{F \in \mathscr{F} \mid F(x) \cap U \neq \phi\}$ where $x \in X$ and $U$ is an open subset of $Y$. Thus let $\mathscr{O}=\{F \in \mathscr{F} \mid F(x) \subset$ $U\}$, and let $H \in \mathcal{O}$. We shall show that there is a $V \in \mathscr{O}$ such that $W(V)[H] \subset \mathcal{O}$. Since $H(x)$ is compact and $U$ is open, there exists a member $V \in \mathscr{O}$ such that $V[H(x)] \subset U$ [1; pg. 199, Th. 33]. Now suppose that $G \in W(V)[H]$; then $(H, G) \in W(V)$ and hence, if $y^{\prime} \in G(x)$, then $\left(H(x), y^{\prime}\right) \cap V \neq \phi$. Therefore there is a $y \in H(x)$ such that $\left(y, y^{\prime}\right) \in V$. Thus $y^{\prime} \in V[y] \subset V[H(x)] \subset U$. That is, $G(x) \subset$ $U$, and so $W(V)[H] \subset U$. Now suppose that $\mathcal{O}=\{F \mid F(x) \cap U \neq \phi\}$, and let $H \in \mathcal{O}$. Then there exists $y \in H(x) \cap \mathcal{O}$ and there exists a $V \in$ of such that $V[y] \subset \mathcal{O}$. If $G \in W(V)[H]$, then $(\mathrm{y}, G(x)) \cap U \neq \dot{\phi}$. Thus there is a $y^{\prime} \in G(x)$ such that $\left(y, y^{\prime}\right) \in V$ and hence, $y^{\prime} \in V[y] \subset \mathcal{O}$. That is, $G(x) \cap U \neq \phi$ and $W(V)[H] \subset \mathcal{O}$. These two results show that the topology of uniform convergence is larger than the topology of pointwise convergence.

We can now use this result to get the following.

THEOREM 2. Let $\mathscr{F}$ be the set of point compact multifunctions on $X$ into $Y$. Then a net $\left\{F_{\alpha}, \alpha \in D\right\}$ converges uniformly to $F \in \mathscr{F}$ if and only if $\left\{F_{\alpha}, \alpha \in D\right\}$ is a Cauchy net, relative to $\mathscr{Y}$, and converges pointwise to $F$.

Proof. That uniform convergence implies pointwise convergence follows from Lemma 1 , and if a net converges uniformly, it is a Cauchy net with respect to $\mathscr{\mathscr { V }}$.

Now suppose that $\left\{F_{\alpha}, \alpha \in D\right\}$ is a Cauchy net with respect to $\mathscr{W}$ and suppose $F_{\alpha} \rightarrow F$ pointwise.

Now let $W(V) \in \mathscr{Y}, V \in \mathscr{Y}$. We need to show that there is a $\beta \in D$ such that $F_{\alpha} \in W(V)[F]$ for all $\alpha \geq \beta$. Let $V^{\prime}$ and $V_{1}$ be closed symmetric members of of such that $V^{\prime} \circ V^{\prime} \subset V_{1} \subset V$. 
Since $\left\{F_{\alpha}, \alpha \in D\right\}$ is Cauchy, there exists a $\beta$ such that $\left(F_{\alpha}, F_{r}\right) \in$ $W\left(V^{\prime}\right)$ for all $\alpha, \gamma \geq \beta$.

Now let $x \in X$, and let $y^{\prime} \in F(x)$. If $V_{0} \in \mathscr{Y}$, then there exists $\beta^{\prime}$ such that $\gamma \geq \beta^{\prime}$ implies that $F_{\gamma}(x) \cap V_{0}\left[y^{\prime}\right] \neq \phi$. Thus there is a net $\left\{y_{\gamma}, \gamma \in D^{\prime}\right\}, y_{\gamma} \in F_{\gamma}(x)$ such that $y_{\gamma} \rightarrow y^{\prime}$. Now if $\alpha>\beta$ and $\gamma>$ $\max \left(\beta^{\prime}, \beta\right)$ then, $\left(F_{\alpha}(x), y_{\gamma}\right) \cap V^{\prime} \neq \phi$. Thus there is a net $\left(y_{\alpha \gamma}, y_{\gamma}\right) \in$ $V^{\prime}, \gamma \in D^{\prime}$ in $F_{2}(x)$. Now if $\left\{y_{\alpha \gamma} \mid \gamma \in D^{\prime}\right\}=A$ is finite there is a $\gamma_{0}$ and a $D_{0}^{\prime}$, a cofinal subset of $D^{\prime}$ such that $\left(y_{\alpha \gamma_{0}}, y_{r}\right) \in V^{\prime}, \quad \gamma \in D_{0}^{\prime}$. Otherwise $A \subset F_{\alpha}(x)$ is infinite and since $F_{\alpha}(x)$ is compact $A$ has a limit point $y_{0} \in F_{\alpha}(x)$. Then in either case there is a $y_{0} \in F_{\alpha}(x)$, such that $\left(y_{0}, y^{\prime}\right) \in$ $V^{\prime}$. We have shown that $\left(F_{\alpha}(x), y^{\prime}\right) \cap V^{\prime} \neq \phi$ for all $x \in X$, where $y^{\prime} \in F(x)$, and for all $\alpha \geq \beta$.

The next step is to show that $(y, F(x)) \cap V^{\prime} \neq \phi$ for all $x$, and all $\alpha \geq \beta$ where $y \in F_{\alpha}(x)$. For this let $x \in X$ and $V_{0} \in \mathscr{V}$ with $V_{0}$ symmetric. Then there exists a $\beta^{\prime}$ such that $F_{\gamma}(x) \subset V_{0}[F(x)]$ for $\gamma>$ $\beta^{\prime}$. Thus there is a net $\left(y, y_{\alpha}^{\prime}\right)$ with $y_{\alpha}^{\prime} \in F_{\gamma}(x)$ and $y_{\gamma} \in F(x), \gamma \in D^{\prime}$.

Now for $y \in F_{\alpha}(x),\left(y, F_{\gamma}(x)\right) \cap V^{\prime} \neq \dot{\phi}$ for $\alpha, \gamma \geq \beta$ and all $x$. Hence, for all $x \in X,\left(y, y_{\gamma}\right) \in V^{\prime} \circ V^{\prime}$ where $y_{\gamma} \in F(x)$. If $y_{0}$ is a limit point of $\left\{y_{r}, \gamma \in D^{\prime}\right\}$ in $F(x)$, then since $V_{1}$ is closed $\left(y, y_{0}\right) \in V_{1} \subset V$ and so (y, $F(x)) \cap V \neq \phi$ for all $x \in X, y \in F_{\alpha}(x), \alpha>\beta$.

These two together imply that $\left(F_{\alpha}, F\right) \in W(V)$ for $\alpha>\beta$ and so $\left\{F_{\alpha}, \alpha \in D\right\}$ converges uniformly to $F$.

DeFinitions. Let $F: X \rightarrow Y$ be a multifunction on a topological space $X$ into a topological space $Y$.

(1) The function $F$ is upper semi-continuous (u.s.c.) if and only if whenever $F(x) \subset V$, an open subset of $Y$, there is an open set $U \subset X$ such that $x \in U$ and $F(U) \subset V$.

(2) The function $F$ is lower semi-continuous (l.s.c.) if and only if whenever $F(x) \cap V \neq \phi, V$ open, there is an open set $U$, such that $x \in U$ and $F\left(x^{\prime}\right) \cap V \neq \phi$ for all $x^{\prime} \in U$.

(3) The function $F$ is continuous if and only if it is both u.s.c. and l.s.c.

THEOREM 3. Suppose the net $\left\{F_{\alpha}, \alpha \in D\right\}$ converges uniformly to $F$. If each $F_{\alpha}$ is u.s.c. (l.s.c., continuous) and if $F$ is point compact, then $F$ is u.s.c. (l.s.c., continuous).

Proof. Let $x \in X$ and $F(x) \subset S$, an open subset of $Y$. Let $V \epsilon$ $\mathscr{O}$ such that $V[F(x)] \subset S$, and $V^{\prime} \in \mathscr{V} \ni V^{\prime} \circ V^{\prime} \subset V$ and let $\beta \in D$ be such that $F_{\alpha} \in W\left(V^{\prime}\right)[F]$ for all $\alpha>\beta$. Now let $\alpha>\beta$ and then $F_{\alpha}(x) \subset V^{\prime}[F(x)]$ (we may assume the $F_{\alpha}(x)$ is contained in the interior of $\left.V^{\prime}[F(x)]\right)$ and so there exists an open set $U \subset X$ such that $F_{\alpha}(U) \subset$ $V^{\prime}[F(x)]$. Also since $\left(F, F_{\alpha}\right) \in W\left(V^{\prime}\right)$ we have $\left(y^{\prime}, F_{\alpha}\left(x^{\prime}\right)\right) \cap V^{\prime} \neq \phi$ for 
all $x^{\prime}$ and $y^{\prime} \in F\left(x^{\prime}\right)$ (in particular this holds for all $x^{\prime} \in U$ ). But then there exists a $y \in F(x)$ such that $\left(y^{\prime}, y\right) \in V^{\prime} \circ V^{\prime} \subset V$ and hence $F\left(x^{\prime}\right) \subset$ $V[F(x)] \subset S$ for all $x^{\prime} \in U$ and thus $F$ is u.s.c.

Now suppose that $F(x) \cap S \neq \phi$, and let $y \in F(x) \cap S$. Further, let $V \in \mathscr{V}$ be such that $V[y] \subset S$ and let $V^{\prime} \in \mathscr{V}$ and $V^{\prime} \circ V^{\prime} \subset V$ (we assume that $V$ and $V^{\prime}$ are symmetric). Let $\beta \in D$ such that $F_{\alpha} \in$ $W\left(V^{\prime}\right)[F]$ for all $\alpha>\beta$. Let $\alpha$ be fixed, $\alpha>\beta$, and then $F_{\alpha}(x) \cap$ $V^{\prime}[y] \neq \phi$. So let $x \in U \subset X$ be an open set such that $F_{\alpha}\left(x^{\prime}\right) \cap V^{\prime}\left[y^{\prime}\right] \neq$ $\phi$ for all $x^{\prime} \in U$, where $y^{\prime} \in F_{\alpha}(x) \subset V^{\prime}[y]$. Now there is a $y^{\prime \prime} \in F\left(x^{\prime}\right)$ such that $\left(y^{\prime \prime}, y^{\prime}\right) \in V^{\prime}$ and hence, $\left(y^{\prime \prime}, y\right) \in V^{\prime} \circ V^{\prime} \subset V$. Hence, $F\left(x^{\prime}\right) \cap$ $S \neq \phi$ for all $x^{\prime} \in U$, and so $F$ is l.s.c.

The above two parts show that if each $F_{\alpha}$ is both u.s.c. and l.s.c., then so is $F$. Consequently if $F_{\alpha}$ is continuous for each $\alpha$, then $F$ is continuous.

Corollary. Let $\left\{F_{\alpha}, \alpha \in D\right\}$ be a net of u.s.c. (l.s.c.) functions into a $T_{2}$-space such that for each $V \in \mathscr{Y}$ there is $a \beta \in D$ such that, $\alpha>\beta$, and $y_{1}, y_{2} \in F_{\alpha}(x)$ implies that $\left(y_{1}, y_{2}\right) \in V$. If $\left\{F_{\alpha}, \alpha \in D\right\}$ converges uniformly to $F$, then $F$ is a continuous single-valued function.

Lemma 4. Suppose that $F: X \rightarrow Y$ is a continuous point compact function on the space $X$ into the regular space $Y$. Let $K \subset X$ be compact, and let $U$ be an open subset of $Y$ such that $F(x) \cap U \neq \phi$ for all $x \in K$. Then there exists a compact set $C \subset U \cap F(K)$ such that $F(x) \cap C \neq \phi$ for all $x \in K$.

Proof. Let $x \in K$ and let $y \in F(x) \cap U$. Then there is an open set $V_{x} \subset Y$ such that $y \in V_{x} \subset V_{x}^{*} \subset U$, and an open set $W_{x} \subset X$ such that $x \in W_{x}$ and if $x^{\prime} \in W$, then $F\left(x^{\prime}\right) \cap V_{x} \neq \phi$. Pick such a $V_{x}$ and $W_{x}$ for each $x \in K$. Thus the family $\mathscr{W}=\left\{W_{x}: x \in K\right\}$ is an open cover of $K$ and so there is a finite subcover, $W_{x_{1}}, \cdots, W_{x_{k}}$. Let $V_{x_{1}}$ be the set corresponding to $W_{x_{1}}$ as above. Then the set $C^{\prime}=\bigcup_{i=1}^{k} V_{x_{i}}^{*}$ $\subset U$ is closed and $F(x) \cap C^{\prime} \neq \phi$ for all $x \in K$. Finally, since $F$ is u.s.c. and point compact, $F(K)$ is compact and so $C=C^{\prime} \cap F(K)$ is the desired set.

REMARK. If we merely require that $F$ be l.s.c. in Lemma 4 , the proof given shows that there is a closed subset $C^{\prime}$ of $U$ such that $F(x) \cap C^{\prime} \neq \phi$ for all $x \in K$.

Lemma 5. Let $\mathscr{F}$ be the family of continuous, point compact functions on a compact space $(X, \mathscr{T})$ into the uniform space $(Y, \mathscr{V})$. Then the topology of uniform convergence is the same as the compact open topology. 
Proof. Let $K \subset X$ be compact and let $\mathscr{O}_{1}, \mathcal{O}_{2}$ be open subsets of $Y$. From [2] the compact open topology is generated by sets of the form $T=\left\{F \in \mathscr{F}: F(K) \subset \mathscr{O}_{1}\right.$ and $F(x) \cap \mathscr{O}_{2} \neq \phi$ for all $\left.x \in K\right\}$. First we shall show that $T$ is open in the topology of uniform convergence. For this let $F \in T$. Since $K$ is compact, $F$ is continuous and $F(x)$ compact, $F(K)$ is compact and so there is a member $U_{1} \in$ $\mathscr{V}$ such that $V_{1}[F(K)] \subset \mathscr{O}_{1} \quad$ Also, by Lemma 4 there is a compact set $C \subset F(K) \cap \mathscr{O}_{2}$ such that $F(x) \cap C \neq \phi$ for all $x \in K$. Let $V_{2}$ be a member of $\mathscr{V}$ such that $V_{2}[C] \subset \mathcal{O}_{2}$, and let $V$ be a symmetric member of $\mathscr{V}$ such that $V \subset V_{1} \cap V_{2}$. Then if $G \in W(V)[F]$, we get $G(K) \cap$ $\mathcal{O}_{1}$ and $G(x) \cap \mathscr{O}_{2} \neq \phi$ for all $x \in K$. Hence $W(V)[F] \subset T$, and so $T$ is open with respect to the topology of uniform convergence.

Now let $V \in \mathscr{V}$ and consider the set $W(V)[F]$. Let $V^{\prime}$ be a closed symmetric member of the uniformity such that $V^{\prime} \circ V^{\prime} \subset V$. If $x \in X$, then, since $F$ is point compact, there exists a finite set $\left\{y_{1}, \cdots, y_{k}\right\} \subset F(x)$ such that $F(x) \subset \bigcup_{1}^{n} V^{\prime}\left[y_{i}\right]^{0}$. Further, by the continuity of $F$, there exists a closed, hence compact, neighborhood $K$ of $x$ such that $F(K) \subset \bigcup_{1}^{k} V^{\prime}\left[y_{i}\right]^{0}$, and $F(z) \cap V^{\prime}\left[y_{i}\right]^{0} \neq \phi$ for all $i=1, \cdots, k$ and $z \in K$. Since $X$ is compact we obtained a finite cover $K_{1}, \cdots, K_{m}$ of $X$ of such sets together with corresponding sets $\bigcup_{1}^{k} V^{\prime}\left[y_{i_{1}}\right]^{0}, \cdots, \bigcup_{1}^{k_{m}} V^{\prime}\left[y_{i_{m}}\right]^{0}$. Set $S_{j}=\bigcup_{1}^{k_{j}} V^{\prime}\left[y_{i_{j}}\right]^{0}$ and define a set $U_{j}$ as follows:

$$
\begin{aligned}
U_{j}= & \left\{G \in \mathscr{F}: G\left(K_{j}\right) \subset S_{j} \text { and } G(x) \cap V^{\prime}\left[y_{i_{j}}\right]^{0} \neq \phi\right. \\
& \text { for all } \left.i_{j} \text { and } x \in K_{j}\right\} .
\end{aligned}
$$

Note that $F \in U_{j}$ for each $j=1, \cdots, m$. Further, let $G \in \bigcap_{1}^{m} U_{j}$ and let $y \in G(x)$ where $x \in K_{j}$. Since $G \in U_{j}, G(x) \subset S_{j}$ and hence, $y \in V^{\prime}\left[y_{i_{j}}\right]^{0}$ for some $y_{i_{j}}$. Also $F(x) \cap V^{\prime}\left[y_{i_{j}}\right] \neq \phi$ follows from the construction of the $K_{j}$ 's. Hence, $(F(x), y) \cap V \neq \phi$. Finally, if $y \in F(x)$ for $x \in K_{j}$, then, since $G(x) \cap V^{\prime}\left[y_{i_{j}}\right]^{0} \neq \phi$, there exists a $y^{\prime} \in G(x)$ such that $\left(y, y^{\prime}\right) \in V^{\prime} \circ V^{\prime}$. Thus $(y, G(x)) \cap V \neq \phi$, and the u.c. topology is contained in the compact open topology. Hence, the lemma follows.

Definition. Let $\mathscr{F}$ be a family of functions on the space $X$ into the uniform space $(Y, \mathscr{V})$ the family $\mathscr{F}$ is equicontinuous at $x \in X$ if and only if for each $V \in \mathscr{Y}$ there is a neighborhood $U$ of $x$ such that for all $F \in \mathscr{F}$ (1) $F(U) \subset V[F(x)]$, and (2) for each $z \in U$, $G(z) \cap V[y] \neq \dot{\phi}$ for all $y \in F(x)$.

Lemma 6. Let $\mathscr{F}$ be a collection of point compact functions on the space $X$ into the uniform space $(Y, \mathscr{Y})$ which is equicontinuous at $x$. Then the pointwise closure of $\mathscr{F}$ in the family of point compact functions on $X$ into $Y$ is also equicontinuous at $x$.

Proof. Let $F$ be a point compact function which is in the 
pointwise closure of $\mathscr{F}$. Let $V$ be a closed member of $\mathscr{V}$ and let $V^{\prime} \in \mathscr{C}$ be closed and symmetric and suppose $V^{\prime} \circ V^{\prime} \subset V$. Let $U$ be a neighborhood of $x$ such that for each $G \in \mathscr{F}, G(U) \subset$ $V^{\prime}[G(x)]$ and $G(z) \cap V^{\prime}[y] \neq \phi$ for $z \in U$ and $y \in G(x)$. Now if $F(U) \not \subset$ $V[F(x)]$, there is a $z \in U$ and $a y \in F(z)$ such that $y \notin V[F(x)]$. Since $F(x)$ is compact, there is a finite set $\left\{y_{1}, \cdots, y_{k}\right\} \subset F(x)$ such that $F(x) \subset$ $\bigcup_{i}^{k} V^{\prime}\left[y_{i}\right]^{0}=S$. Further there is an open set $T$ such that $y \in T$ and $T \cap V\left[y_{i}\right]=\phi$ for each $i$. Now set $W(S)=\{H: H(x) \subset S\}$ and $W(T)=$ $\{H: H(z) \cap T \neq \phi\}$. Then $W=W(S) \cap W(T)$ is a pointwise open set containing $F$ such that $W \cap \mathscr{F}=\phi$. This is a contraction and so we conclude that $F(U) \subset V[F(x)]$. A similar argument will show that $F(z) \cap V[y] \neq \phi$ for all $y \in F(x)$ and $z \in U$. Hence the lemma follows.

We say that the family $\mathscr{F}$ is equicontinuous in case it is equicontinuous at each point. Then if $\mathscr{F}$ is equicontinuous, each member of $\mathscr{F}$ is 1.s.c., and if each member of $\mathscr{F}$ is point compact, thene ach member of $\mathscr{F}$ is u.s.c. and hence, each member of $\mathscr{F}$ is continuous.

Let $\mathscr{F}$ be a family of functions from the topological space $X$ into the topological space $Y$. A topology $\mathscr{T}$ on $\mathscr{F}$ is said to be jointly continuous (j.c.) if and only if the function $P: \mathscr{F} \times X \rightarrow Y$ defined by $P(F, x)=F(x)$ is continuous.

LEMma 7. If $\mathscr{F}$ is an equicontinuous collection of point compact functions, then the pointwise topology for $\mathscr{F}$ is jointly continuous.

Proof. First we shall show that $P$ is u.s.c. For this suppose that $F(x) \subset W$ where $W$ is an open subset of $Y$ and $(F, x) \in \mathscr{F} \times X$. Since $F(x)$ is compact, there is a symmetric $V$ in the uniformity such that $V \circ V[F(x)] \subset W$, and such that $F(x) \subset \bigcup V[y]^{\circ}=S$ for $y \in$ $F(x)$. Since $\mathscr{F}$ is equicontinuous, there is a neighborhood $U$ of $x$ such that $G(U) \subset V[G(x)]$ for all $G \in \mathscr{F}$. Further, let $T=\{G \in \mathscr{F}: G(x) \subset S\}$. Then $(F, x) \in T \times U$ and $P(T \times U) \subset W$. A similar argument shows $P$ is l.s.c. and so the pointwise topology is jointly continuous.

CoRollary. If $F$ is an equicontinuous family of point compact functions on the compact space $X$ into the uniform space $(Y, \mathscr{V})$, then the u.c. topology, the pointwise topology and the compact open topology are all the same.

Proof. This follows from Lemmas 5 and 7 together with Propositions 6 and 7 of [2].

We need one more lemma before stating one of the main theorems of this paper.

Lemma 8. If $\mathscr{F}$ is compact relative to a j.c. topology $\mathscr{T}$, and if each member of $\mathscr{F}$ is point compact, then $\mathscr{F}$ is equicontinuous. 
Proof. Let $V \in \mathscr{Y}$; the uniformity for $Y$; let $V^{\prime}$ be a symmetric member of $\mathscr{V}$ such that $V^{\prime} \circ V^{\prime} \subset V$, and let $x \in X$. Since $P$ is continuous, we can find a neighborhood $W(F)$ for each $F \in \mathscr{F}$ and a neighborhood $U(F)$ of $x$ such that there is a finite subset $\left\{y_{1}, \cdots, y_{k}\right\}$ of $F(x)$ and such that for $G \in W(F), G(z) \subset \bigcup_{1}^{k} V^{\prime}\left[y_{i}\right]$, and $G(z) \cap$ $V^{\prime}\left[y_{i}\right] \neq \phi$ for each $y_{i}$. Then there is a finite subcover $W\left(F_{1}\right), \cdots$, $W\left(F_{n}\right)$ of $\mathscr{F}$ with corresponding sets $U\left(F_{1}\right), \cdots, U\left(F_{n}\right)$. Let $U=$ $\bigcap_{i=1}^{n} U\left(F_{j}\right)$. Then if $G \in \mathscr{F}, G \in W\left(F_{j}\right)$ for some $j$, and if $z \in U$, then $G(z) \subset \bigcup_{i}^{k} V^{\prime}\left[y_{i}\right]$ where $y_{1} \in F_{j}(x)$. Thus, since $G(x) \cap V^{\prime}\left[y_{i}\right] \neq \phi$, for each $y \in G(z)$ there is a $y^{\prime} \in G(x)$ such that $\left(y, y^{\prime}\right) \subset V^{\prime} \circ V^{\prime} \subset V$ and so $G(z) \subset V[G(x)]$ for all $z \in U$. On the other hand let $y \in G(x)$. Since $G(x) \subset \bigcup_{1}^{k} V^{\prime}\left[y_{i}\right]$ and $G(z) \cap V^{\prime}\left[y_{i}\right] \neq \phi$ for all $y$, we get $G(z) \cap V[y] \neq$ $\phi$. Hence, $\mathscr{F}$ is equicontinuous at $x$, and the lemma follows.

Now by combining the above results with Theorem 3 in [2] we get the following Ascoli Theorem.

THeorem 9. Let $\mathscr{C}$ be the set of all continuous, point compact functions on a compact regular space into a $T_{2}$, uniform space. Let $\mathscr{C}$ have the topology of uniform convergence. Then a subset $F \subset \mathscr{C}$ is compact if and only if

(i) $\mathscr{F}$ is closed in $\mathscr{C}$,

(ii) $F[x]=\cup\{F(x): F \in \mathscr{F}\}$ has compact closure for each $x \in X$, and

(iii) $\mathscr{F}$ is equicontinuous.

We can extend many of the above results in the following way. Let $\mathscr{A}$ be a family of subsets of $X$. Then in the definition of $W(V)$ replace $x \in X$ by $x \in A$ for some $A \in \mathscr{A}$. Then generate a uniformity by these sets. This gives us the topology of uniform convergence on members of $\mathscr{A}$. (If $\mathscr{A}=\{X\}$, there is no difference, and if $\mathscr{A}$ is all singletons we get pointwise convergence). In particular if $\mathscr{A}$ is the set of compact sets then we obtain the topology of uniform convergence on compacta. Then if we use the topology of uniform convergence on compacta in place of the u.c. topology, we can obtain results analogous to Theorem 9 for functions on locally compact spaces.

\section{REFERENCES}

1. J. L. Kelley, General Topology, Van Nostrand, New York, N. Y., 1955.

2. R. E. Smithson, Topologies on Sets of Relations, Journal Nat. Sci. and Math. Government College (Lahore) to appear.

Received April 15, 1969.

UNIVERSITY OF WYOMING 



\section{PACIFIC JOURNAL OF MATHEMATICS}

\section{EDITORS}

H. SAMELSON

Stanford University

Stanford, California 94305

C. R. HobBY

University of Washington

Seattle, Washington 98105
J. DugundjI

Department of Mathematics

University of Southern California

Los Angeles, California 90007

RICHARD ARENS

University of California

Los Angeles, California 90024

\section{ASSOCIATE EDITORS}

E. F. BECKENBACH

B. H. NeUMaNN

F. WOLF

K. YoshidA

\section{SUPPORTING INSTITUTIONS}

UNIVERSITY OF BRITISH COLUMBIA

CALIFORNIA INSTITUTE OF TECHNOLOGY

UNIVERSITY OF CALIFORNIA

MONTANA STATE UNIVERSITY

UNIVERSITY OF NEVADA

NEW MEXICO STATE UNIVERSITY

OREGON STATE UNIVERSITY

UNIVERSITY OF OREGON

OSAKA UNIVERSITY
UNIVERSITY OF SOUTHERN CALIFORNIA STANFORD UNIVERSITY

UNIVERSITY OF TOKYO

UNIVERSITY OF UTAH

WASHINGTON STATE UNIVERSITY UNIVERSITY OF WASHINGTON

$\stackrel{*}{*} \stackrel{*}{*} \stackrel{*}{*}$ AMERICAN MATHEMATICAL SOCIETY
NAVAL WEAPONS CENTER 


\section{Pacific Journal of Mathematics}

Vol. 39 , No. 1

May, 1971

Charles A. Akemann, A Gelfand representation theory for $C^{*}$-algebras ....

Sorrell Berman, Spectral theory for a first-order symmetric system of

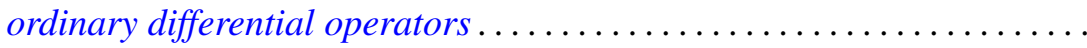

Robert L. Bernhardt, III, On splitting in hereditary torsion theories ........

J. L. Brenner, Geršgorin theorems, regularity theorems, and bounds for determinants of partitioned matrices. II. Some determinantal identities ..........................................

Robert Morgan Brooks, On representing $F^{*}$-algebras .............. 51

Lawrence Gerald Brown, Extensions of topological groups........... 71

Arnold Barry Calica, Reversible homeomorphisms of the real line ........ 79

J. T. Chambers and Shinnosuke Oharu, Semi-groups of local Lipschitzians in

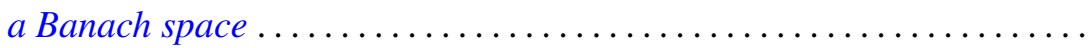

Thomas J. Cheatham, Finite dimensional torsion free rings .............

Byron C. Drachman and David Paul Kraines, A duality between

transpotence elements and Massey products ...................

Richard D. Duncan, Integral representation of excessive functions of a

Markov process ......................................

George A. Elliott, An extension of some results of Takesaki in the reduction

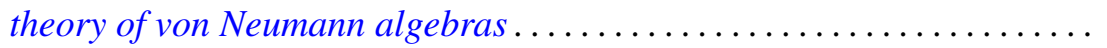

Peter C. Fishburn and Joel Spencer, Directed graphs as unions of partial

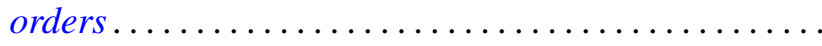

Howard Edwin Gorman, Zero divisors in differential rings ...

Maurice Heins, A note on the Löwner differential equations...

Louis Melvin Herman, Semi-orthogonality in Rickart rings. .

David Jacobson and Kenneth S. Williams, On the solution of linear G.C.D.

equations

Michael Joseph Kallaher, On rank 3 projective planes ... . .

Donald Paul Minassian, On solvable $O^{*}$-groups ...........

Nils Øvrelid, Generators of the maximal ideals of $A(\bar{D})$

Mohan S. Putcha and Julian Weissglass, A semilattice decomposition into

semigroups having at most one idempotent ............

Robert Raphael, Rings of quotients and $\pi$-regularity ....

J. A. Siddiqi, Infinite matrices summing every almost periodic sequence. .

Raymond Earl Smithson, Uniform convergence for multifunctions ...

Thomas Paul Whaley, Mulitplicity type and congruence relations in

universal algebras...

Roger Allen Wiegand, Globalization theorems for locally finitely generated modules... 